\title{
Proposal of upgrade of the ATLAS muon trigger in the Barrel - End Cap transition region with RPCs
}

\author{
L. Massa* on behalf of the ATLAS Muon collaboration \\ University of Bologna and INFN (Italy) \\ E-mail: lorenzo.massa@cern.ch
}

\begin{abstract}
This report presents a project for the upgrade of the Level-1 muon trigger in the Barrel - End Cap transition region $(1.0<|\eta|<1.3)$ of the ATLAS detector with RPC chambers. The ATLAS Level-1 muon trigger rate is dominated by fake triggers in the End Cap region $(|\eta|>1)$ caused by charged particles originating from secondary interactions downstream of the interaction point. After the LHC upgrade forseen for 2018, the Level-1 muon trigger rate would saturate the allocated bandwidth unless new measures are adopted to improve the rejection of fake triggers. ATLAS is going to improve the trigger selectivity in the region $|\eta|>1.3$ with the New Small Wheel detector upgrade. To obtain a similar trigger selectivity in the Barrel - End Cap transition region, it is proposed to add new RPC chambers at the edge of the inner layer of the Barrel muon spectrometer. These chambers will be based on a three layer structure with thinner gas gaps and electrodes with respect to the ATLAS standard. New front-end electronics, integrating fast TDC capabilities will be used. A preliminary study based on 2012 data demonstrates that the new system could reject more than $90 \%$ of the fake triggers while maintaining high trigger efficiency. This will allow keeping a relatively low momentum threshold while matching the rate requirements of future LHC running.
\end{abstract}

Technology and Instrumentation in Particle Physics 2014,

2-6 June, 2014

Amsterdam, the Netherlands

\footnotetext{
* Speaker.
} 


\section{Introduction}

\subsection{The ATLAS muon spectrometer}

ATLAS [1] is a multipurpose experiment at the Large Hadron Collider (LHC), which covers almost completely the whole solid angle, using a large number of sub-detectors. In particular, the muon spectrometer, situated in the outer region of ATLAS, is fundamental to detect and reconstruct the muons, which are the only charged particles which are expected not to be stopped by the calorimeter. The trajectory and the momentum of such particles are measured using toroidal magnetic fields and two types of precision chambers: Monitored Drift Tubes (MDT) and Cathode Strip Chambers (CSC). The ATLAS muon spectrometer is also equipped with faster chambers that are used by the trigger: the Barrel region (modulus of pseudorapidity $|\eta|<1$ ) is covered by Resistive Plate Chambers (RPC) while the End Cap region ( $|\eta|>1)$ is covered by Thin Gap Chambers (TGC).

The ATLAS trigger system is based on a hardware based level (L1) and a software based higher-level trigger (HLT). It reduces the rate from $40 \mathrm{MHz}$ to about $200 \mathrm{~Hz}$. The Level 1 Muon Trigger is based on RPC and TGC hits and defines the Regions of Interest (ROI) that are used to seed the HLT.

In the coming years the LHC will have an intense upgrade program: starting from 2018 there will be the so called Run-3, with a center-of-mass energy of $\sqrt{s}=13 \sim 14 \mathrm{TeV}$, an instantaneous luminosity up to $L=3 \cdot 10^{34} \mathrm{~cm}^{-2} \mathrm{~s}^{-1}$ and $25 \mathrm{~ns}$ of bunch crossing interval. In order to perform well in these conditions, the trigger and tracking systems of the muon spectrometer in the $|\eta|>1.3$ region will be upgraded replacing the inner layer of the End Cap with the New Small Wheel (NSW) [2]. Another proposal is the upgrade of the muon trigger in the Barrel - End Cap transition region using RPCs, which is the project this article is about.

\subsection{High rate in transition region}

During Run-3, the estimated total rate of the Level-1 single muon trigger (with transverse momentum $p_{T}>20 \mathrm{GeV}$ ) will rise up to $57.6 \mathrm{kHz}$, if no measures are taken, while ATLAS can allocate only $25 \mathrm{kHz}$ for muon triggers out of a total Level-1 bandwidth of $100 \mathrm{kHz}$ [3]. Figure 1 shows the $\eta$ distribution of the ROIs of the Level-1 single muon trigger [2]. There is great abundance of ROIs in the $|\eta|>1$ region, while the population of the reconstructed muons (which are drawn in darker blue) is almost flat. Hence, most of these ROIs are background, mainly low- $p_{T}$ protons generated in toroids and shieldings of the spectrometer. The trigger request in the NSW will reduce the rate in the $|\eta|>1.3$ region, while there will be still a high background rate in the transition region between Barrel and End Cap $(1.0<|\eta|<1.3)$. The rate coming from the transition region in runs of $25 \mathrm{~ns}$ of bunch crossing interval is about $21.9 \%$, which corresponds to $12.6 \mathrm{kHz}$ at $\sqrt{s}=13$ $\mathrm{TeV}$ and $L=3 \cdot 10^{34} \mathrm{~cm}^{-2} \mathrm{~s}^{-1}$.

The rate can be reduced requiring a coincidence between the End Cap trigger and the passage through an inner plane. The Barrel is divided in 16 sectors in azimuthal angle $\phi$, divided into small and large sectors. The small sectors contain the coils of the Barrel toroidal magnetic field. For the large sectors of the spectrometer this inner plane is covered by the TGCs in the inner layer of the End Cap (EI), while in the small sectors the EI chambers do not extend in the transition region. The proposal is to add RPCs to BIS 7-8 chambers, which are the inner Barrel MDT chambers that cover the transition region (figure 2, left). 


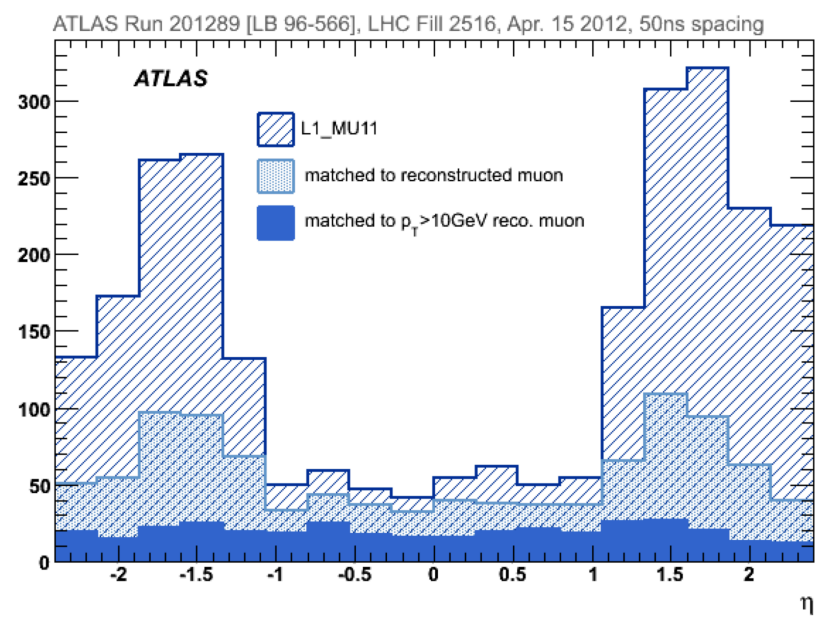

Figure 1: $\eta$ distribution of Level-1 muon ROIs $\left(p_{T}>10 \mathrm{GeV}\right)$ with the distribution of the subset matched to an offline reconstructed muon with $p_{T}>3 \mathrm{GeV}$ or $p_{T}>10 \mathrm{GeV}$ [2].

The geometrical coverage in the range $1<\eta<1.3$ is shown in figure 2 on the right.
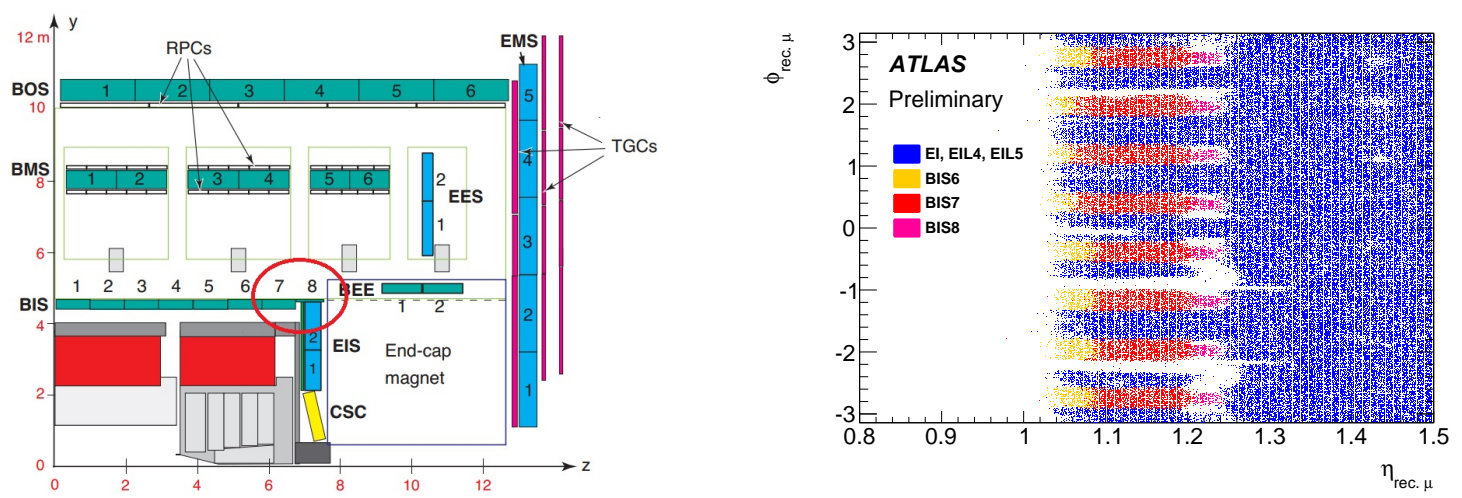

Figure 2: On the left: the small sectors of the Barrel of the ATLAS muon spectrometer. The red circle highlights the BIS7-8 chambers, where the RPCs are proposed to be added. On the right: $\eta-\phi$ distribution of reconstructed muons ( $p_{T}>20 \mathrm{GeV}$ and associated to an End Cap trigger) associated to segments in the inner End Cap Chambers (EI) (in blue) and in the BIS chambers (all other colors) [4].

The coverage of the TGCs in large sectors is limited by some holes due to the rails and the cryo-lines of ATLAS. The small sectors are covered by the BIS chambers, in particular by the BIS7 which occupies a large part of the transition region. The total coverage of the designed trigger is estimated to be about $83.5 \%$ of the transition region.

\section{Performance studies}

An analysis has been made with 2012 data, requiring the End Cap trigger and emulating the RPC hits on the proposed chambers using MDT track segments. The study is based on both standard runs with 50ns of bunch crossing interval and special runs with $25 \mathrm{~ns}$ of bunch crossing interval 
that present background conditions closer to Run-3. The ROIs are considered matched to a muon if the reconstructed muon has a $p_{T}>20 \mathrm{GeV}$ and a $\Delta R$ (ROI, reconstructed muon) $<0.1$ (where $\left.\Delta R=\sqrt{\Delta \eta^{2}+\Delta \phi^{2}}\right)$. The MDT track segments used to emulate the RPC hits are required to point to the interaction point, to lie in a sector which is compatible with $\phi$ of the ROI and to lie within a certain $\Delta \eta=\eta_{\text {segment }}-\eta_{R O I}$ from the ROI.
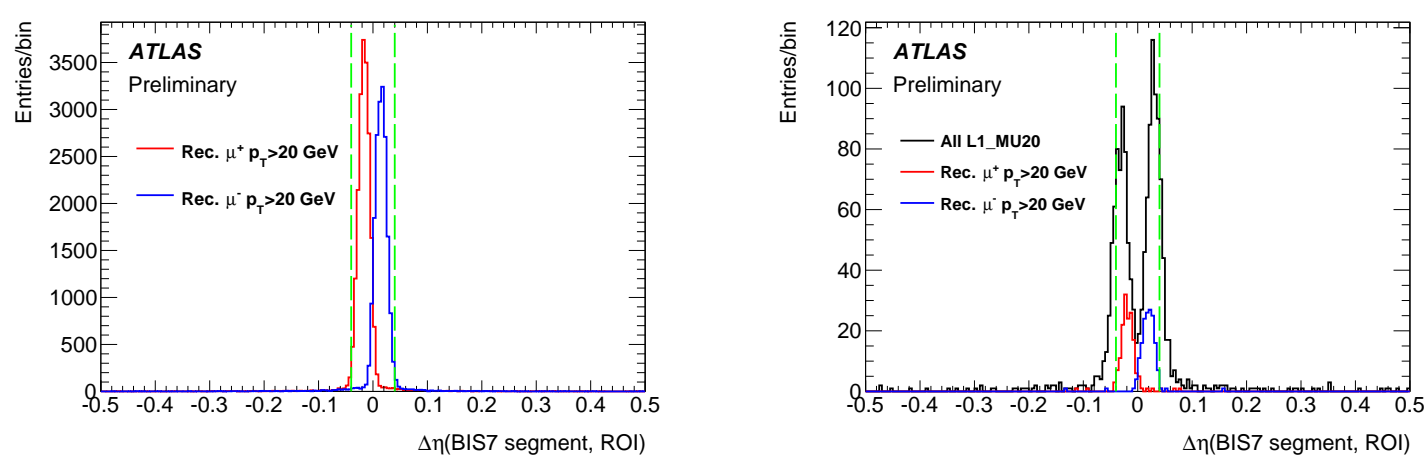

Figure 3: The distribution of $\Delta \eta$ between the track segments inside the BIS7 chamber and the ROIs of Level1 single muon trigger $\left(p_{T}>20 \mathrm{GeV}\right)$ associated to the reconstructed muons [4]. On the left the distribution is obtained with runs at $50 \mathrm{~ns}$; on the right the distribution is obtained with runs at $25 \mathrm{~ns}$. On the right the distribution obtained using all the ROIs is also drawn (in black).

\subsection{The effects of a $\Delta \eta$ cut}

Figure 3 shows the distribution of $\Delta \eta$ for the BIS7 chamber. On the left the distribution is obtained with standard runs at $50 \mathrm{~ns}$ and associating the ROIs to the reconstructed muons; on the right the distribution is obtained with special runs at $25 \mathrm{~ns}$. With these runs it has been possible to perform also the same calculation using all the ROIs and obtaining a distribution which includes the background. The signal is concentrated in a region of $|\Delta \eta|<0.04$, hence a $\Delta \eta$ criterium in the algorithm of the trigger would allow rejecting a higher background fraction. In fact, as can be seen in figure 4 , a $\Delta \eta$ cut of 0.04 leads to a further rejection of almost $\sim 30 \%$ of the events. All the events which are rejected are low $p_{T}$ particles, which correspond to background.

\subsection{Results}

The $\eta$ distribution of ROIs in End Caps before and after applying the request of RPC hits in the proposed chambers is shown in figure 5. The request of segments in the BIS and EI chambers reduces drastically the number of events in the transition region.

The performance of the designed trigger has been evaluated studying the following quantities. The efficiency is the ratio between the reconstructed muons triggered by End Cap associated with segments in the proposed chambers and all the reconstructed muons triggered by End Cap $\left(\frac{\left.N_{\text {MuReco }} \text { EndCap \& }(B I S|| E I)\right)}{N_{\text {MuReco }}(\text { EndCap })}\right)$.

The rate fraction is the ratio between the ROIs in the End Cap associated with segments in the proposed chambers and all the ROIs in the End Cap $\left(\frac{\left.N_{R O I}(\text { EndCap\&(BIS } \mid E I)\right)}{N_{R O I}(\text { EndCap })}\right)$. 


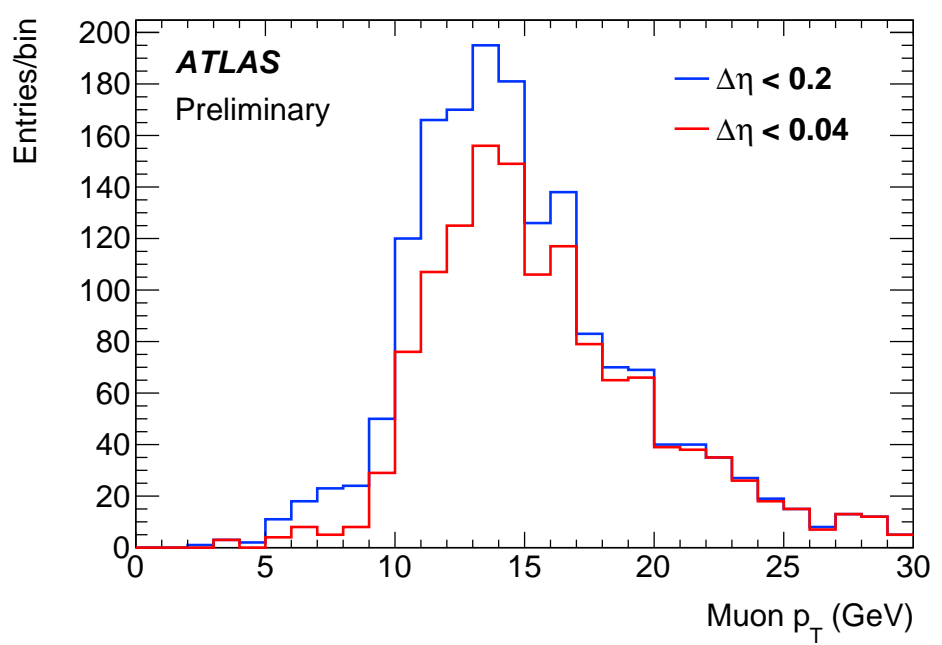

Figure 4: Distribution of the transverse momentum of the reconstructed muons obtained with different cuts in the difference $\Delta \eta$ between the segments in the inner plane and the Level-1 single muon ROIs $\left(p_{T}>20\right.$ $\mathrm{GeV}$ ) [4]. The distribution obtained with $\Delta \eta<0.2$ is drawn in blue, while the one obtained with $\Delta \eta<0.04$ is drawn in red.

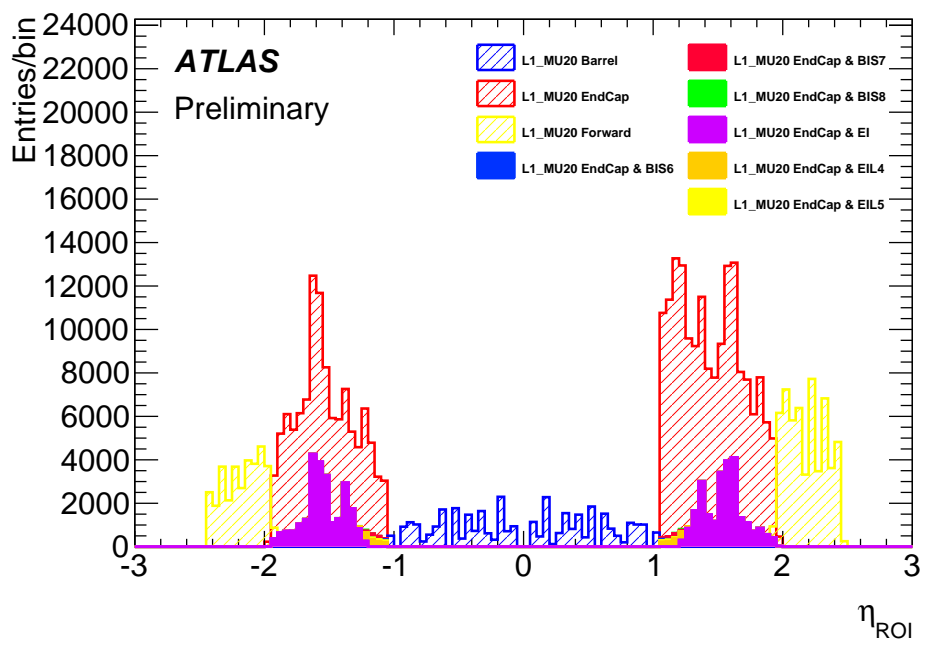

Figure 5: The $\eta$ distribution of the ROIs ( $p_{T}>20 \mathrm{GeV}$ ) in runs with $25 \mathrm{~ns}$ of bunch crossing interval [4]. The hatched distribution is obtained with the current End Cap trigger, while the fully colored one is obtained requiring the passage through the EI or the BIS chambers.

The background fraction is the ratio between the ROIs in the End Cap associated with segments in the proposed chambers but not to any reconstructed muons and all the ROIs in the End Cap $\left(\frac{N_{R O I}(\text { EndCap\& }(B I S|| E I) \& ! M u R e c o)}{N_{R O I}(\text { EndCap })}\right)$.

The results are shown in table 1 . The values have been calculated for three different cases: 1) asking the association between ROIs and segments of the BIS+EI chambers for all the ROIs of the transition region; 2) asking the association between ROIs and segments of the EI chambers only in EI acceptance and making no requests on other ROIs; 3) asking the association between ROIs 


\begin{tabular}{|c|c|c|c|}
\hline \hline & $\begin{array}{c}\text { BIS+EI } \\
\text { everywhere }\end{array}$ & $\begin{array}{c}\text { EI } \\
\text { in EI acceptance }\end{array}$ & $\begin{array}{c}\text { BIS+EI } \\
\text { in BIS+EI acceptance }\end{array}$ \\
\hline Efficiency & $80.1 \%$ & $99.8 \%$ & $98.9 \%$ \\
Rate Fraction & $6.8 \%$ & $54.5 \%$ & $35.2 \%$ \\
Background Fraction & $5.5 \%$ & $53.5 \%$ & $34.1 \%$ \\
\hline \hline
\end{tabular}

Table 1: Efficiency, rate fraction and background fraction calculated in the transition region for three different cases. In the first column the segments inside BIS and EI are requested in association to all ROIs of the transition region; in the second (third) column the EI (BIS+EI) segments are requested only in association to ROIs in the EI (BIS+EI) acceptance, and no requiremenrs are made on other ROIs.

and segments of the BIS+EI chambers only in BIS+EI acceptance and making no requests on other ROIs. In the latter case there is an important reduction of the rate and background fraction with a small decrease of the efficiency.

\subsection{Interaction with the Tile calorimeter}

It is important to notice that the RPC BIS7-8 project is not the only one which has been studied in order to reduce the high fake trigger rate in transition region: the Tile calorimeter will be used starting from 2015 [3] to confirm the End Cap triggers using the sum of calorimeter cell signal. The requirement of a coincidence with the Tile Calorimeter will provide high efficiency $(\sim 97 \%)$ and an expected surviving rate fraction of $\sim 18 \%$. In combination with the New Small Wheel, the Tile calorimeter will reduce the total rate of Level-1 single muon trigger (with $p_{T}>20 \mathrm{GeV}$ ) from 57.6 $\mathrm{kHz}$ to about $12 \mathrm{kHz}$, while it has been estimated that the combination of the New Small Wheel and the BIS7-8 would reduce the rate to about $\sim 10 \mathrm{kHz}$. An opportune combination of Tile, BIS and End Cap chambers could lead to a high efficiency trigger $(\sim 97 \%)$ with a surviving rate fraction of at most $\sim 10 \%$ and higher robustness against pile up effects.

\section{Mechanical layout}

The mechanical layout of the proposed new trigger is challenging, because of the limited available space in the detector. In order to cope with these difficulties, it has been proposed to replace the BIS7 and BIS8 MDT chambers with an integrated chamber holding a new type of small MDT [5] (with diameter of $15 \mathrm{~mm}$ instead of $30 \mathrm{~mm}$ of standard MDTs) and new RPC [6] in the same envelope of the old MDT chambers, as shown in figure 6. This layout leads to some further advantages. First of all, the new small MDTs have better rate capability, which is particularly important in this region, where the highest rate on the MDTs is expected. Finally, the redesign of the chamber allows obtaining the optimal geometrical coverage of the trigger.

\subsection{The new Resistive Plate Chamber design}

A new tecnology of smaller RPCs [6] with respect to the old ones [7] has been studied, which make possible a basic chamber thickness of about $48 \mathrm{~mm}$. The new chamber will be a three-layer detector operating with a 2/3 majority configuration. The new gas gaps will be of $1 \mathrm{~mm}$ instead of $2 \mathrm{~mm}$ of the standard RPCs, and will be equipped with a new front end electronics that will reduce 


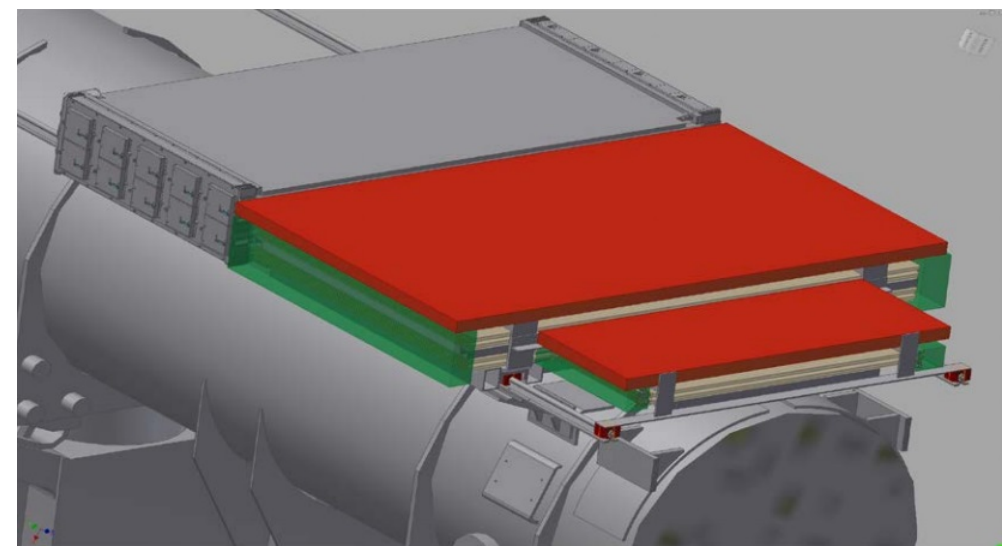

Figure 6: The proposed layout of an integrated chamber holding the small MDT (green and gray) and RPC (in red) in the same envelope of the old MDT chambers. The small MDT chamber is made of a single piece covering the area of BIS7 an BIS8, the RPC is instead split in two.

the delivered charge, leading to higher rate capability, improved aging and better time resolution. Also the electrode plates and the supports will be thinner with respect to the usual RPCs. The $\eta$ coordinate will be read through strips of $\sim 2 \mathrm{~cm}$ pitch. Two options are under consideration for the measurement of the $\phi$ coordinate: with a layer of strips othogonal to the $\eta$ strips, as in the standard ATLAS RPCs, or exploiting the signal propagation time along the strips, using a double side readout of the $\eta$ strips. A full custom charge-to-voltage amplifier in BJT technology has been developed for small RPCs, which require an extremely good Signal-to-Noise $S / N$ ratio, radiation hardness and the possibility to match a low impedance transmission line [8]. Tests performed with cosmic rays demonstrate that, with the new amplifier and standard $2 \mathrm{~mm} \mathrm{RPCs}$, it is possible to obtain full efficiency at a lower applied voltage, reducing the average charge per count from 20 $\mathrm{pC} /$ count to $3 \mathrm{pC} /$ count [9] (figure 7). This leads to an improvement of detector rate capability and ageing. Tests performed with a 1+1 $\mathrm{mm}$ bi-gap RPC (with gaps of $2 \mathrm{~mm}$ divided in two sub-gaps of $1 \mathrm{~mm}$ by a thin metal layer) and the new amplifier have shown a total charge per avalanche of $\sim$ $2 \mathrm{pC} /$ count [10].
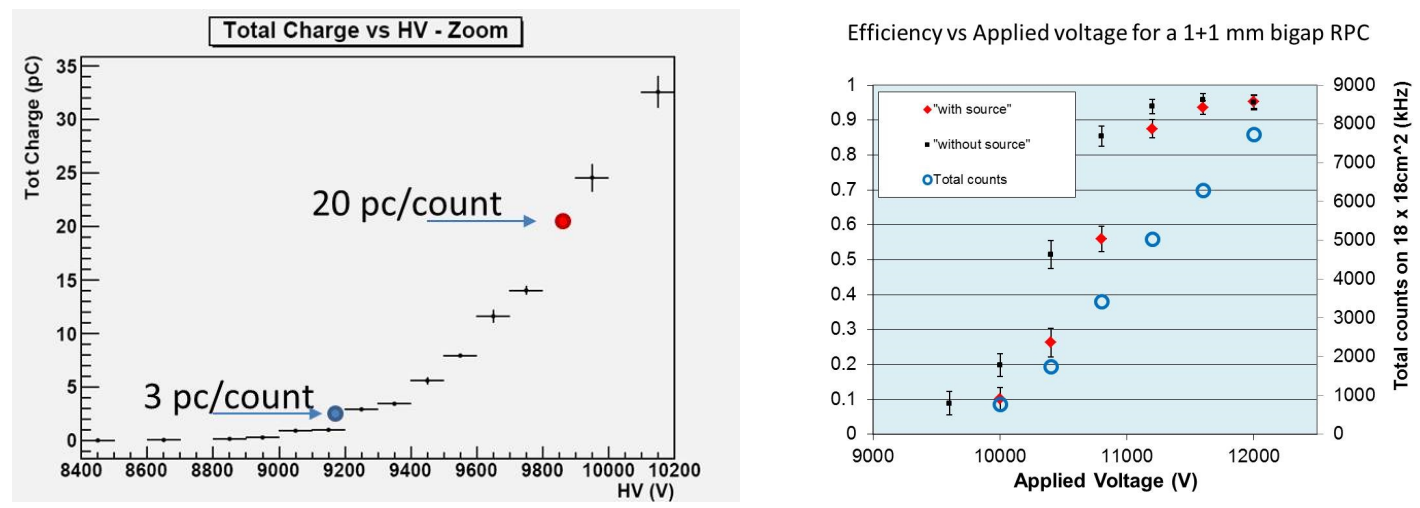

Figure 7: On the left: avalanche charge as a function of applied voltage, using the new amplifier and standard RPCs [9]. On the right: efficiency vs applied voltage for a $1+1 \mathrm{~mm}$ bigap RPC, showing full efficiency at a counting rate of $11 \mathrm{kHz} / \mathrm{cm}^{2}$, using the new amplifier [10]. 


\section{Conclusions}

An upgrade of the ATLAS muon trigger in the Barrel - End Cap transition region with RPCs has been proposed in order to reduce the fake trigger rate. Performance studies made with 2012 data show that the proposed upgrade reduces significantly the rate, keeping almost all the signal. A combination with the Tile calorimeter, BIS7-8 and End Cap TGCs allows a full coverage of the transition region with a selective trigger. The challenge of the integration of new chambers in an already existing system, with a limited amount of available space, has motivated the development of an integrated chamber with new small MDTs with a diameter of $15 \mathrm{~mm}$ for precise tracking and a triplet of new RPCs. The proposed new RPCs will have gas gaps of $1 \mathrm{~mm}$ and a new front end amplifier which together lead to an improvement of the rate capability.

\section{References}

[1] The ATLAS Collaboration, The ATLAS Experiment at the CERN Large Hadron Collider, JINST 3 (2008) S08003.

[2] The ATLAS Collaboration, New Small Wheel Technical Design Report, CERN-LHCC-2013-006, ATLAS-TDR-020, [https: / / cds . cern. ch/record/1552862].

[3] The ATLAS Collaboration, Technical Design Report for the Phase-I Upgrade of the ATLAS TDAQ System, CERN-LHCC-2013-018, ATLAS-TDR-023,

[https://cds.cern.ch/record/1602235].

[4] The ATLAS Muon Collaboration, L1 Rate reduction by adding RPCs to BIS, [https: / / atlas.web. cern. ch/Atlas/GROUPS/PHYSICS/MUON/PublicPlots / 2014 /ATL-COM-MUON-2014-008/].

[5] B. Bittner et al., Development of fast high-resolution muon drift-tube detectors for high counting rates, Nucl. Instr. and Meth. A 628 (2011) 154.

[6] R. Santonico, A new generation of RPCs to be used as muon trigger detectors at the super-LHC, Technology and Instrumentation in Particle Physics 2011, Chicago, 9-14 June 2011, [http://indico.cern.ch/event/102998/session/16/contribution/427].

[7] R. Santonico and R. Cardarelli, Development of resistive plate counters, Nucl. Instr. and Meth. A 187 (1981) 377.

[8] R. Cardarelli et al., Performance of RPCs and diamond detectors using a new very fast low noise preamplifier, JINST 8 (2013) P01003.

[9] R. Cardarelli, A fast electronics for RPC based precision tracking muon trigger at high luminosity LHC, XI workshop on Resistive Plate Chamber and Related Detectors, LNF, 5-10 February 2012, [https: / / agenda.infn.it/contributionDisplay.py? contribId=67\&session $I d=5 \& \operatorname{conf} I d=3950]$.

[10] L. Paolozzi et al., Performance of a new generation RPCs for particle physics at colliders of the next generation, poster at 13th Vienna Conference of Instrumentation, Vienna University of Technology, 11-15 February 2013, [http: / / indico. cern. ch/event/186337/contribution/216]. 